increased and LEF $10 \mathrm{mg}$ or LEF dose increase to $20 \mathrm{mg}$ or alternative steroid sparing agent in case of LEF ineffectiveness recommended). The number of relapses and a cumulative $\mathrm{GC}$ dose during follow-up were recorded.

Results: Between July 2014 and December 2016 we identified 76 (65.8\% female, median (IQR) age 73.7 (66.1-78.8) years) new GCA cases with a follow-up of at least 48 weeks (median (IQR) $75^{51-104}$ weeks). $30 / 76$ patients $(39.5 \%$ ) received LEF at W12 ("LEF" group), the others continued with GC only. During the followup 22 patients relapsed, 4 in "LEF" group (13.3\%) and 18 (39.1\%) in "GC only" group. The difference was statistically significant $(p=0.02$; NNT $3.9(95 \% \mathrm{Cl} 2.2-$ $17.4)$ ). Furthermore, $17 / 30$ GCA cases $(56.7 \%)$ in "LEF" group managed to stop GC at W48 (with 1 relapse (5.9\%) shortly afterwards), but none in GC only group. Patients tolerated LEF relatively well. Adverse events (AEs) were usually mild. 8/ 30 patients $(26.7 \%$ ) discontinued LEF (1 due to ineffectiveness and 7 due to one/ more AEs - hair loss developed in 4/7 cases, diarrhoea in 2/7 patients, weight loss in 2/7 cases, and elevated transaminases in 1/7). The occurrence of infections requiring antibiotics and/or hospital admission was lower in LEF group compared to "GC only" group (10\% vs. $26.5 \%$ ).

Conclusions: We found in our prospective observational study in GCA a steroid sparing action and a rather good tolerability of LEF.

Disclosure of Interest: None declared

DOI: 10.1136/annrheumdis-2018-eular.2751

\section{SAT0519 INTERSTITIAL LUNG DISEASE IN ANCA-ASSOCIATED VASCULITIS PATIENTS:COMPARISON WITH IDIOPATIC PULMONARY FIBROSIS AND INTERSTITIAL PNEUMONIA WITH AUTOIMMUNE FEATURES}

A. Abril ${ }^{1}$, M. Kwon ${ }^{2}$, C. Rojas ${ }^{3}$, A. Khoor ${ }^{4}$, I. Mira-Avendano ${ }^{2} .{ }^{1}$ Rheumatology; ${ }^{2}$ Pulmonary medicine; ${ }^{3}$ Radiology; ${ }^{4}$ Pathology, Mayo Clinic, Jaksonville, FI, USA

Background: Patients with ANCA-positive vasculitis may develop interstitial lung disease (ILD), it is an uncommon but increasingly recognised manifestation. Clinical characteristics and prognosis are not well known in these patients. The largest report to date is from East Asia describing microscopic polyangitis (MPA) as the most common association with ILD.

Objectives: To describe the cliniccal manifestatiions and response to therapy of patients with AAV and ILd compared with patients with idiopathic pulmonary fibrosis (IPF) and interstitial pneumonia with autoimmune features (IPAF)

Methods: We conducted a retrospective 10 year chart review at the Mayo Clinic Florida, including patients with confirmed diagnoses of both AAV and ILD done by expert rheumatology and pulmonology clinical evaluations. Clinical characteristics of ANCA-ILD in 24 patients were compared to 29 patients with idiopathic pulmonary fibrosis (IPF) and 22 patients with interstitial pneumonia with autoimmune features (IPAF), which were confirmed by applying the IPF diagnostic criteria based on the most recent ATS guidelines and IPAF criteria defined by Fischer and colleagues, respectively.

Results: We identified 24 ANCA-ILD patients. 14 patients had MPA, 12 patients had granulomatosis with polyangitis (GPA), and 2 patients had eosinophilic granulomatosis with polyangitis (EGPA). $54 \%$ were female and mean age was 70 . In half of the ANCA-ILD patients, vasculitis presented prior to ILD, mainly MPA, 36\% of patients presented with ILD first, most of them with GPA. The rest presented with ILD and vasculitis at the same time. Usual interstitial pneumonia (UIP) was the most common radiographic pattern. Honeycombing was more common in MPA compared to GPA patients. Ground glass opacity was present in 5 (63\%) of GPA and in $5(36 \%)$ of MPA patients. Most MPA patients had positive anti-MPO antibody and p-ANCA. Only one GPA patient had positive anti-MPO antibody and two were $\mathrm{p}$-ANCA positive. The majority of the GPA patients were positive for antiproteinase-3 antibody and c-ANCA. The mainstay of treatment was corticosteroids. Rituximab was used in 14 patients. The decline in functional vital capacity (FVC) and diffusing capacity (DLCO) was most marked in IPF group, followed by ANCA-ILD and then the IPAF group ( $\triangle \mathrm{FVC},-0.5,-0.5$, and $0.3 \mathrm{~L} / \mathrm{s} ; \Delta \mathrm{DLCO}$, $-3.7,-3.6$, and -0.1 , respectively). In a similar manner, survival was poorest in IPF, followed by ANCA-ILD and was best in the IPAF group.

Conclusions: This is, to our knowledge, the largest case series of clinically confirmed AAV with ILD in North America. Sizable number of GPA with c-ANCA positive patients presenting concomitant ILD is a novel observation for the clinical characteristics of ANCA-ILD that contradicts previous epidemiology of ANCAILD. Our data is also of value by adding prognostic information in an era of newer therapeutics, such as rituximab. In addition, the intermediate prognosis of ANCAILD, between IPF and IPAF, is very interesting especially after the new classification IPAF.

Disclosure of Interest: None declared

DOI: 10.1136/annrheumdis-2018-eular.1255

\section{SAT0520 ANTI-IL6-RECEPTOR TOCILIZUMAB IN REFRACTORY UVEITIS ASSOCIATED TO EXTRAOCULAR MANIFESTATIONS IN PATIENTS WITH BEHÇET'S DISEASE. MULTICENTER STUDY OF 11 PATIENTS}

B. Atienza-Mateo ${ }^{1}$, J.L. Martín-Varillas ${ }^{1}$, V. Calvo-Río ${ }^{1}$, E. Beltrán², L. Martinez ${ }^{3}$, E. Valls ${ }^{4}$, M. Hernandez ${ }^{5}$, A. Atanes ${ }^{6}$, M. Cordero ${ }^{7}$, J.M. Nolla ${ }^{8}$, C. Carrasco ${ }^{9}$, J. Loricera ${ }^{1}$, N. Palmou-Fontana ${ }^{1}$, E. Pons ${ }^{1}$, M.C. González-Vela ${ }^{1}$, R. DemetrioPablo ${ }^{1}$, M.A. González-Gay ${ }^{1}$, R. Blanco ${ }^{1} .{ }^{1}$ Rheumatology, Anatomic Pathology and Ophthalmology, Hospital Universitario Marqués de Valdecilla. IDIVAL, Santander, ${ }^{2}$ Rheumatology, Hospital General Universitario de Valencia; ${ }^{3}$ Ophthalmology; ${ }^{4}$ Rheumatology, Hospital Peset, ${ }^{5}$ Ophthalmology, Hospital General Universitario de Valencia, Valencia; ${ }^{6}$ Rheumatology, Hospital Universitatio de A Coruña, A Coruña; ${ }^{7}$ Rheumatology, Hospital de León, León; ${ }^{8}$ Rheumatology, Hospital Universitari de Bellvitge, Barcelona; ${ }^{9}$ Rheumatology, Hospital de Mérida, Mérida, Spain

Objectives: To assess the efficacy of Tocilizumab (TCZ) in refractory uveitis associated to extraocular manifestations due to Behcet's disease (BD).

Methods: Multicenter study of patients with BD refractory to standard systemic treatment (conventional immunosuppressive drugs and/or anti-TNF- $\alpha$ agents).

Results: We studied 11 patients ( 7 men/4 women) (20 affected eyes); mean age $38.4 \pm 20.4$ years. Uveitis was bilateral in 9 patients. The pattern of ocular involvement was: panuveitis ( $n=8$; with retinal vasculitis in 4$)$, anterior uveitis $(n=2)$ and posterior uveitis $(n=1)$. Cystoid macular oedema (CME) was present in 7 patients. The clinical course was recurrent $(n=7)$ or chronic $(n=4)$. Apart from the visual complications, at TCZ onset the following extraocular manifestations were present: oral and/or genital ulcers $(n=7)$, arthritis $(n=4)$, folliculitis/pseudofolliculitis $(n=4)$, erythema nodosum $(n=2)$, livedo reticularis $(n=1)$, intestinal affection $(n=1)$, and neurological involvement $(n=2)$.

Before TCZ, they had received systemic corticosteroids, conventional immunosuppressive drugs and biologic agents, adalimumab $(n=8)$, infliximab $(n=4)$, golimumab $(n=3)$, canakinumab $(n=1)$, or etanercept $(n=1)$. TCZ was used in monotherapy or combined with conventional immunosuppressive drugs at $8 \mathrm{mg} /$ $\mathrm{kg} / \mathrm{iv} / 4$ weeks $(\mathrm{n}=10)$ or $162 \mathrm{mg} / \mathrm{sc} /$ week $(n=1)$. TCZ yielded rapid and maintained improvement in all ocular parameters (TABLE). After a mean follow-up of 9.5 \pm 8.05 months using TCZ, all patients experienced ocular improvement, with complete remission in 8 of them. However, TCZ was only effective in 3 of the patients with extraocular manifestations. This biologic agent had to be withdrawn in 2 cases, 1 due to a severe infusion reaction and 1 due to arthritis impairment.

Abstract SAT0520 - Table 1. Epidemiological and clinical characteristic of the sample.

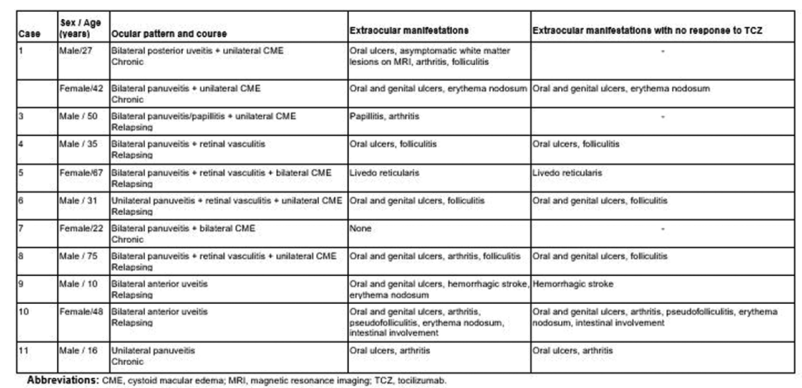

Abbreviations:

CME, cystoid macular oedema; MRI, magnetic resonance imaging; TCZ, tocilizumab.

Conclusions: TCZ appears to be useful in highly refractory BD-related uveitis. However, there are controversial results regarding its efficacy in the treatment of extraocular manifestations of $\mathrm{BD}$.

\section{REFERENCES}

[1] Santos-Gómez M, Calvo-Río V, Blanco R, Beltrán E, Mesquida M, Adán A. The effect of biologic therapy different from infliximab or adalimumab in patients with refractory uveitis due to Behcet's disease: results of a multicenter open label study. Clin Exp Rheumatol 2016;34(6 Suppl 102):S34S40.

[2] Calvo-Río V, de la Hera D, Beltran-Catalan E, Blanco R, Hernandez M., Martinez-Costa L. Tocilizumab in uveitis refractory to other biologic drugs: a study of 3 cases and a literature review. Clin Exp Rheumatol 2014;32 (Suppl 84):S54-S57.

[3] Calvo-Río V, Santos-Gomez M, Calvo I, et al. Anti-IL6-R Tocilizumab for Severe Juvenile Idiopathic Arthritis-Associated Uveitis Refractory to antiTNF therapy. A multicenter study of 25 patients. Arthritis Rheumatol 2016;69:668-75. 